\title{
Multipath Interference Cancellation in MIMO Mobile Cellular system
}

\author{
Nazia Parveen, \\ Research scholar JNTU, Hyderabad Andhra Pradesh, India. \\ nazia_ifreyahoo.co.in \\ D.S. Venkateswarlu \\ Progressive Engineering College, Cheekati Midi, Hyderabad \\ Andhra Pradesh, India. \\ dronamraju_sveyahoo.com
}

\begin{abstract}
Wireless network widely used today include, cellular network, wireless mesh network (WMNs), wireless local area network and personal area network. The increasing demand for these networks has turned spectrum into a precious resource. For this reason, there is always a need for methods to pack more bits $/ \mathrm{Hz}$. A particular solution is to use multiple antenna at both transmitter and receiver side. Such a system is called multiple-input multiple-output (MIMO) system. In real propagation environment of cellular communication, channel reuse will cause the co-channel interference which will degrade the MIMO channel performance. The interference cancellation (IC technology) can handle efficiently the cochannel interference. In this paper comparison computer simulation for zero forcing successive interference cancellation (ZF-SIC) minimum mean square error (MMSE equalizer) and maximum likelihood $(M L)$ for two transmitting and two receiving antenna is shown.
\end{abstract}

KEYWORDS

MIMO, co-channel interference, spatial multiplexing diversity scheme.

\section{INTRODUCTION}

Wireless communication is highly challenging due to complex time varying propagation medium. If we consider a wireless link with one transmitter and one receiver the transmitted signal that is launched into wireless environment arrives at the receiver along a number of diverse paths referred to as multipath. These paths occur from scattering and rejection of radiated energy from objects (buildings, hills, trees etc.) and each path a different time-varying delay, angle of arrival and signal amplitude. As a consequence the received signal can vary as a function of frequency, time and space. These variations are referred as fading and cause detoriation of the system quality. Further more wireless channel suffer from co-channel interference (CCI) from other cells that share the same frequency channel, leading to distortion of the desired signal and also low system performance. Therefore, wireless system must be designed to mitigate fading and interference to guarantee a reliable communication. A successful method to improve reliable communication over a wireless link is to use multiple antennas.

The next generation wireless systems are required to have high voice quality as compared to current cellular mobile radio standards and provide high bit data rate services (upto $2 \mathrm{mbits} / \mathrm{sec}$ ). The next generation systems are supposed to have better quality and coverage, be more powerful and bandwidth efficient and be deployed in diverse environment. [1][2] 


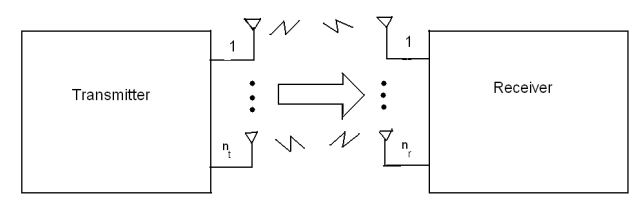

Fig.1. MIMO system

A MIMO wireless system is a communication link for which we have multiple antennas at the transmitter as well as multiple antennas at the receiver as depicted in Fig.1. The principal goal of MIMO technology is to improve either the quality (BER) or the data rate of the communication by means of adequate signal processing techniques at both ends of the system. The capacity can increase linearly with the number of antennas when using MIMO system. MIMO can obtain both multiplexing gain and diversity gain thus significantly increasing the system capacity as well as improving the reliability of the wireless link. The advantages of MIMO communication are as follows.

1. Array gain: Due to the use of multiple antennas, we have an average increase in the SNR at the receiver due to the inherent combining effect of the transmitted signals. This gain is available through multiple antennas processing at the transmitter or the receiver or both.

2. Diversity gain: By using the benefits and knowledge of the well known receiver diversity theory we can develop systems using multiple antennas at the transmitter (MISO/MIMO) to obtain transmit diversity. Diversity techniques rely on transmitting the signal over multiple (ideally) independent fading paths (time/frequency/space) that are often called diversity branches. The probability that all diversity branches will be on a deep fade at the same time is small, making the communication link more robust. Spatial diversity is favoured over time or frequency diversity because it does not incur in transmission time or bandwidth expenditure when inserting redundancy, i.e. it is spectrally more efficient.

3. Multiplexing gain: It has been proved that MIMO channels offer a linear increase in the capacity or transmission rate for the same bandwidth with no additional power usage. Spatial Multiplexing(SM) gain is realized by transmitting independent sub-streams of data signal from each antenna. Under favourable conditions the receiver can identify the different sub-streams and combine these to yield the original data stream.

4. Interference suppression: By taking advantage of the spatial domain it is possible to differentiate between the spatial signatures of the desired signal and co-channel interference signal to reduce the interference.

Space -time coding stands as a technique that exploits the advantages given by MIMO channel in order to solve traffic capacity bottle necks in actual SISO communication system and consist on the co-ordinated encoding of both temporal and special domains in order to introduce redundancy and thus provide diversity. Space-time code has very appealing property is the so called orthogonal space-time block code (OSTBC). [3][4]. OSTBC achieves full diversity and guarantees a decoupled maximum likelihood (ML) detection of the different transmitted symbol.

In the mobile telephone area, MIMO has been considered in the 3GPP and 3GPP2 working groups of the ITU. IMT-2000 standard organization in fact the Alamouti coding scheme which is a space-time strategy for coding is already part of both WCDMA and CDMA 2000 standards, and the bell laboratories space-time layer architecture, (BLAST) which is another signalling scheme has also been considered to work in UMTS. In the WLAN field, multi antenna technology has demonstrated to boost the performance of IEEE $802.11 \mathrm{a} / \mathrm{b} / \mathrm{g}$ system and the IEEE 802.11n task group is responsible for standardizing the development of the next generation wireless system. Intel wimaz is also embracing MIMO concepts to bring wireless high speed connection to entire metropolitan areas. 
In the cellular environment there will be channel reuse and therefore co-channel interference from other cells exist and as a result the performance will be degraded. So it is necessary to reject the co-channel interference. This co-channel interference are short peaks and can be handled using interference cancellation technology. In this paper we will introduce MIMO technology in detail and then analyse the co-channel interference and its cancellation methods for cellular systems. We will introduce a scheme to reject the co-channel interference using ZF-SIC (Zero forcing successive interference cancellation), MMSE (Minimum Mean square error) Equaliser and ML (Maximum Likelihood) equalizer and present the simulation results.

\section{MIMO SYSTEM MODEL}

MIMO systems are composed of three main elements, namely the transmitter (TX), the channel $(H)$, and the receiver $(\mathrm{RX})$. In this paper, $\mathrm{N}_{\mathrm{t}}$ is denoted as the number of antenna element at the transmitter and $\mathrm{N}_{\mathrm{r}}$ is denoted as the number of elements at the receiver. Fig. 2 depicts the Physical MIMO channel. The channel with $\mathrm{N}_{\mathrm{r}}$ outputs and $\mathrm{N}_{\mathrm{t}}$ inputs is denoted as $\mathrm{N}_{\mathrm{r}} * \mathrm{~N}_{\mathrm{t}}$ matrix.

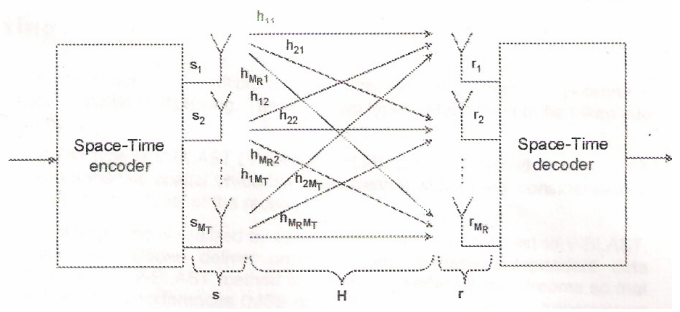

Fig.2. Physical MIMO channel

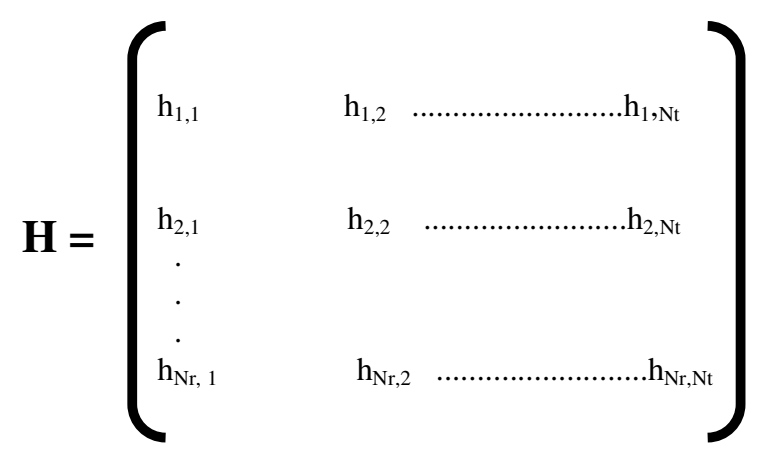

Where each entry $h_{i, j}$ denotes the attenuation and phase shift(transfer function) between the $j^{\text {th }}$ transmitter and the $\mathrm{i}^{\text {th }}$ receiver.

The MIMO signal model is described as

$$
\mathrm{r}=\mathrm{Hs}+\mathrm{n}
$$

where' $r$ ' is the received vector of size $\mathrm{N}_{\mathrm{r}}{ }^{*} 1, \mathrm{H}$ is the channel matrix of size $\mathrm{N}_{\mathrm{r}}{ }^{*} \mathrm{~N}_{\mathrm{t}}$, 's' is the transmitted vector of size $\mathrm{N}_{\mathrm{t}}^{*} 1$, and ' $\mathrm{n}$ ' is the noise vector of size $\mathrm{N}_{\mathrm{r}}{ }^{*} 1$.Each noise element is typically modelled as independent identically distributed (i.i.d) white Gaussian noise [5], [6] with variance $\mathrm{N}_{t} /(2 . \mathrm{SNR})$ [3]. To prevent correlation due to spacing they are typically spaced at least $\lambda c / 2$ where $\lambda c$ is the wavelength of the carrier frequency [5]. The second reason 
correlation can occur is due to lack of multipath components. It is for this reason that rich multipath is desirable in MIMO system. The multipath effect can be interpreted by each receive antenna being in a different channel. For this reason the rank of MIMO channel is defined as the number of independent equations offered. It is important to note that

$$
\operatorname{Rank}(\mathrm{H}) \leq \min \left(\mathrm{N}_{\mathrm{r}}, \mathrm{N}_{\mathrm{t}}\right)
$$

And therefore the maximum number of streams that a MIMO system can support is upper bounded by $\min \left(\mathrm{N}_{\mathrm{r}}, \mathrm{N}_{\mathrm{t}}\right)$.

\section{MIMO TECHNIQUES}

Current MIMO system includes MISO and SIMO system that uses MIMO technique to improve the performance of wireless system can be divided into two kinds. One is spatial multiplexing which provides a linear capacity gain in relation to the number of transmitting antenna and the other is spatial diversity schemes which can reduce the BER and improve the reliability of the wireless link.

\subsection{SPATIAL MULTIPLEXING}

The transmission of multiple data stream over more than one antenna is called spatial multiplexing. In this technology multiple antennas are used at both the ends to increase the bit rate in wireless radio link without additional power or bandwidth consumption. It offers a linear increase in spectral efficiency with the number of antennas. There are two types which have to be taken into account. The first is the V- BLAST (Vertical Bell Laboratories Layered SpaceTime) which transmit spatial un-coded data streams without any consideration in equalising the signal at the receiver. In V-BLAST algorithm, instead of decoding all transmitted signal at the same time, we first decode the 'strongest' signal, then subtract this strongest signal from the received signal and proceed to decode the strongest signal of the remaining transmit signal and so on. Other algorithms include

1. Zero forcing $(\mathrm{ZF})$ receiver

2. Minimum Mean square error (MMSE)algorithm

3. Maximum Likelihood (ML) receiver algorithm.

The ML receiver algorithm can yield the best performance. The second one is realized by space time codes. In contrast to V-BLAST space time codes deliver orthogonal \& thereby independent data streams. The V- BALST method is not able to separate the stream so that multi stream interferences can appear. This makes the transmission unsteady \& forward error coding is not always able to resolve this issue. The detection of space time coded signal is based on a simple linear process and achieves reasonable results.

\subsection{DIVERSITY SCHEMES}

To improve the link reliability we are using diversity schemes. Spatial diversity improves the signal quality and achieves higher signal to noise ratio at the receiver side. Two kinds of spatial diversities are considered, Transmitter diversity and Receiver diversity. There are two famous space time coding schemes. Space time block code (STBC) and Space time trellis code (STTC). STTC generally offers better performance than STBC. To utilise transmitter diversity the so called Almouti space time code can be applied. It achieves full diversity and works with one receiving antenna. Receiver diversity can be used through more receiving antenna than transmitting antenna and a proper combining algorithm. Switched combining or maximum ratio combining are the two examples of algorithms. This works independently on the type of diversity if the channel matrix is known. 


\section{CO-CHANNEL INTERFERENCE}

The co-channel interference is one of the major limitations in cellular telephone network. In the case of cellular network such as $3 \mathrm{G}$ or beyond $3 \mathrm{G}(4 \mathrm{G})$, the co-channel interference is caused by the frequency reuse. Our main idea is to reject the co- channel interference in MIMO cellular systems.

\subsection{INTERFERENCE CANCELLATION TECHNIQUES}

To eliminate co-channel interference we use two techniques. One is successive interference cancellation and the other is parallel interference cancellation.

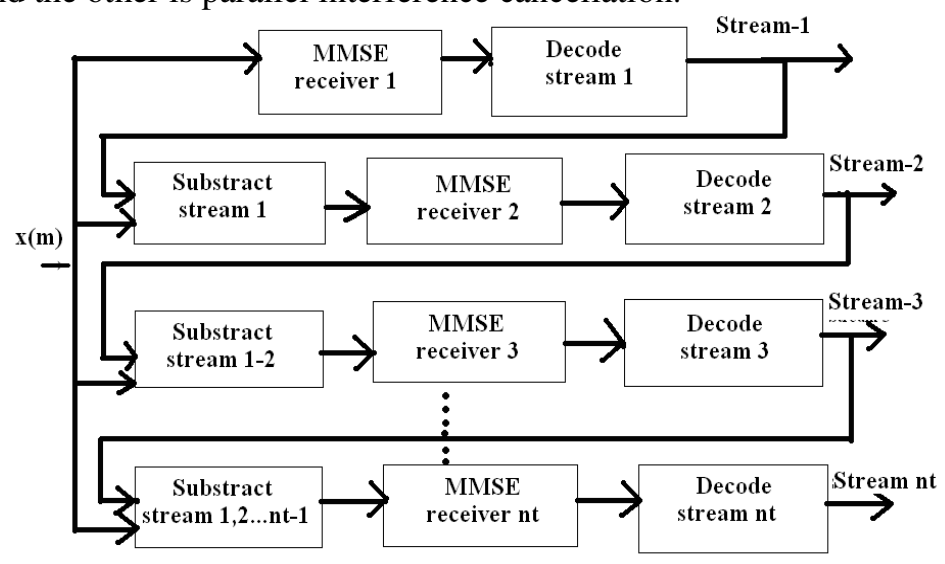

Fig.3 SIC using MMSE receiver

Fig. 3 shows the block diagram of successive interference cancellation using MMSE receiver. The idea was first proposed by Viterbi. We use de-correlator to decode the data stream $\mathrm{x}_{1}(\mathrm{~m}) \&$ then substract of $f$ this decoded stream from the received vector. If the first stream is successfully decoded then the second decorrelator has to deal only with stream $\mathrm{x}_{3}$----------- $\mathrm{x}_{\mathrm{n}}$ as interference. Sincex $x_{1}$ has been correctly subtracted off. This process is continued until the final decorrelator does not have to deal with any interference from the other data streams. After the subtraction process is completed, the residual signal would contain less interference. The algorithm repeats this subtraction process with other user until the weakest user signal has been detected.

\subsection{PARALLEL INTERFERENCE CANCELLATION (PIC)}

Successive interference cancellation will increase one additional bit delay per subtraction process. But in PIC cancellation estimates and subtract out all the interference for each user in parallel in order to reduce the time delay.

PIC works better than SIC when all the users are received with equal strength, this is the difference between these two types of interference cancellation. Here the user's symbols are estimated in a parallel manner. PIC cancellation is also known as multi stage process where in each stage an attempt is made for each user to completely cancel the interference caused by all other users. 


\section{BPSK MODULATION FOR (SISO) SYSTEM.}

The theoretical equation for bit error rate (BER) with Binary Phase Shift Keying (BPSK) modulation scheme in Additive White Gaussian Noise (AWGN) channel is derived first. The BER results are obtained using MATLAB simulation scripts which show good agreement with the derived theoretical results.

With Binary Phase Shift Keying (BPSK), the binary digits 1 and 0 may be represented by the analog levels $+\sqrt{ } E_{b}$ and- $\sqrt{ } E_{b}$ respectively. The system model is as shown in the Fig.4

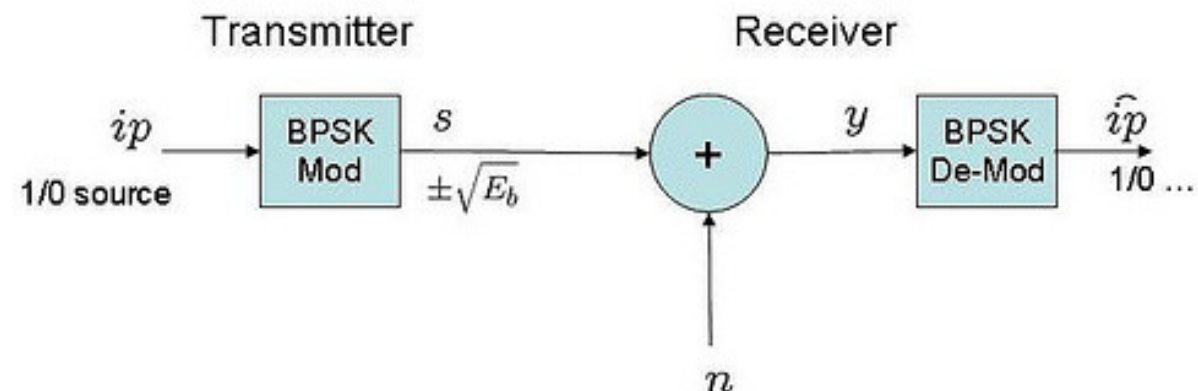

Fig.4 Simplified block diagram with BPSK transmitter-receiver

\subsection{Channel Model}

The transmitted waveform gets corrupted by noise $\mathrm{n}$, typically referred to as Additive White Gaussian Noise (AWGN).

Additive: As the noise gets 'added' (and not multiplied) to the received signal

White: The spectrum of the noise if flat for all frequencies.

Gaussian: The values of the noise $\mathrm{n}$ follows the Gaussian probability distribution function, $p(x)=\frac{1}{\sqrt{2 \pi \sigma^{2}}} e^{-(x-\mu)^{2}}$ with $\mu=0$ and $\sigma^{2}=\frac{N_{0}}{2}$.

\section{Computing the probability of error}

The received signal,

$\mathrm{y}=\mathrm{s}_{1}+\mathrm{n}$ when bit 1 is transmitted and

$\mathrm{y}=\mathrm{s}_{0}+\mathrm{n}$ when bit 0 is transmitted.

The conditional probability distribution functions (PDF) of y for the two cases are:

$p\left(y s_{0}\right)=\frac{1}{\sqrt{\pi N_{0}}} e^{\frac{-\left(y+\sqrt{S_{b}}\right)^{2}}{N_{0}}}$ 
$p\left(y \mid s_{1}\right)=\frac{1}{\sqrt{\pi N_{0}}} e^{\frac{-\left(y-\sqrt{B_{b}}\right)^{2}}{N_{0}}}$
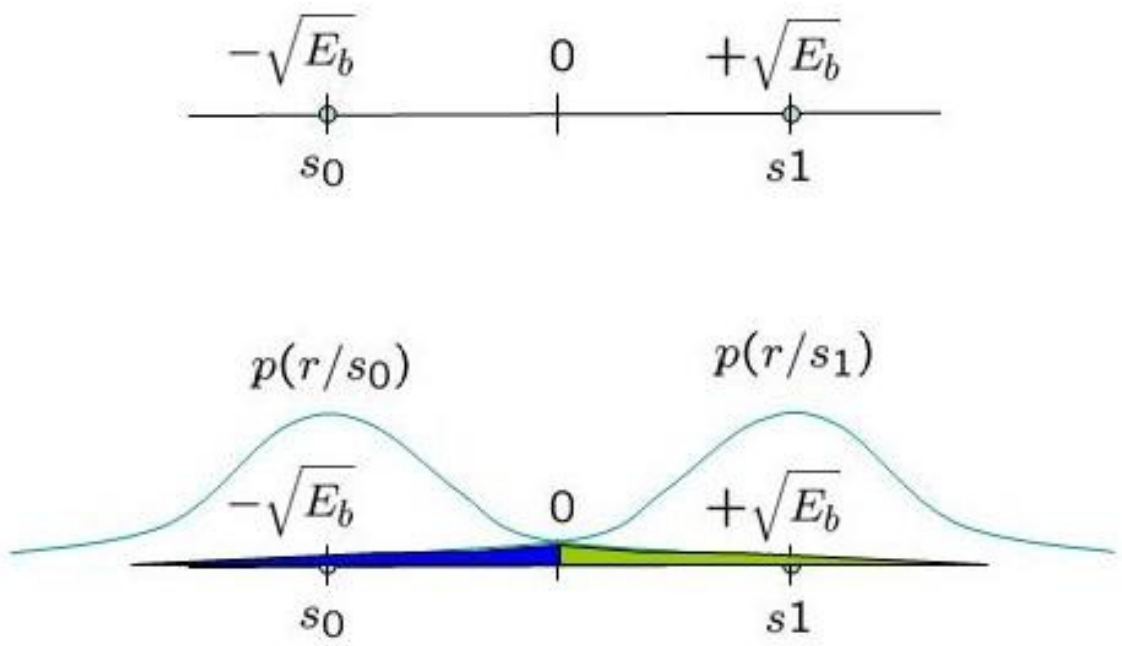

Fig.5 Conditional probability density function with BPSK modulation

Assuming that $s_{0}$ and $s_{1}$ are equally probable i.e., the threshold 0 forms the optimal decision boundary.

- $\quad$ if the received signal is greater than 0 , then the receiver assumes $\mathrm{s}_{1}$ was transmitted.

- $\quad$ if the received signal is less than or equal to 0 , then the receiver assumes $s_{0}$ was transmitted.

i.e.

$y>0 \Rightarrow s_{1}$ and

$y \leq 0 \Rightarrow s_{\mathrm{C}}$.

\section{1) Probability of error given $s_{1}$ was transmitted}

With this threshold, the probability of error given $\mathrm{s}_{1}$ is transmitted is (the area in blue region):

$p\left(e \mid s_{1}\right)=\frac{1}{\sqrt{\pi N_{0}}} \int_{-\infty}^{0} e^{\frac{-\left(y-\sqrt{B_{b}}\right)^{2}}{N_{0}}} d y=\frac{1}{\sqrt{t}} \int_{\frac{g_{b}}{N_{0}}}^{\infty} z^{2} d z=\frac{1}{2} e r f c\left(\sqrt{\frac{B_{b}}{N_{\mathrm{C}}}}\right)$

where, 
$\operatorname{erf}(x)=\frac{2}{\sqrt{7}} \int_{x}^{\infty} e^{-x^{2} d x}$ Is the complementary error function.

\section{2) Probability of error given $s_{0}$ was transmitted}

Similarly the probability of error given $\mathrm{s}_{0}$ is transmitted is (the area in green region):

$$
p\left(e \mid s_{0}\right)=\frac{1}{\sqrt{\pi N_{0}}} \int_{\mathrm{C}}^{\infty} e^{\frac{-\left(y+\sqrt{R_{b}}\right)^{3}}{N_{0}}} d y=\frac{1}{\sqrt{\pi}} \int_{\sqrt{\frac{E_{b}}{N_{0}}}}^{\infty} e^{2} d z=\frac{1}{2} \operatorname{erfc}\left(\sqrt{\frac{E_{b}}{N_{0}}}\right)
$$

\section{3) Total probability of bit error}

$\mathrm{P}_{\mathrm{b}}=\mathrm{p}\left(\mathrm{s}_{1}\right) \mathrm{p}\left(\mathrm{els} \mathrm{s}_{1}\right)+\mathrm{p}\left(\mathrm{s}_{0}\right) \mathrm{p}\left(\mathrm{els} \mathrm{s}_{0}\right)$

Given that we assumed that $\mathrm{s}_{1}$ and $\mathrm{s}_{0}$ are equally probable i.e. $\mathrm{p}\left(\mathrm{s}_{1}\right)=\mathrm{p}\left(\mathrm{s}_{0}\right)=1 / 2$, the bit error probability is,

$P_{b}=\frac{1}{2} \operatorname{erfc}\left(\sqrt{\frac{E_{0}}{N_{0}}}\right)$

\section{Simulation Algorithm}

(a) Generation of random BPSK modulated symbols +1 's and -1's

(b) Passing them through Additive White Gaussian Noise channel

(c) Demodulation of the received symbol based on the location in the constellation

(d) Counting the number of errors

(e) Repeating the same for multiple $\mathrm{E}_{\mathrm{b}} / \mathrm{N}_{0}$ value.

\section{2. $2 \times 2$ MIMO channel}

In a $2 \times 2$ MIMO channel, probable usage of the available 2 transmit antennas can be as follows: 1. Consider that we have a transmission sequence, for example $\left\{\mathrm{x}_{1}, \mathrm{x}_{2}, \mathrm{x}_{3} \ldots \mathrm{x}_{\mathrm{n}}\right\}$

2. In normal transmission, we will be sending $x_{1}$ in the first time slot, $x_{2}$ in the second time slot, $\mathrm{x}_{3}$ and so on.

3. However, as we now have 2 transmit antennas, we may group the symbols into groups of two. In the first time slot, we send $x_{1}$ and $x_{2}$ from the first and second antenna. In second time slot, we send $x_{3}$ and $x_{4}$ from the first and second antenna; send $x_{5}$ and $x_{6}$ in the third time slot and so on.

4. Notice that as we are grouping two symbols and sending them in one time slot, we need only $\mathrm{n} / 2$ time slots to complete the transmission - data rate is doubled.

5. This forms the simple explanation of a probable MIMO transmission scheme with 2 transmit antennas and 2 receive antennas. 


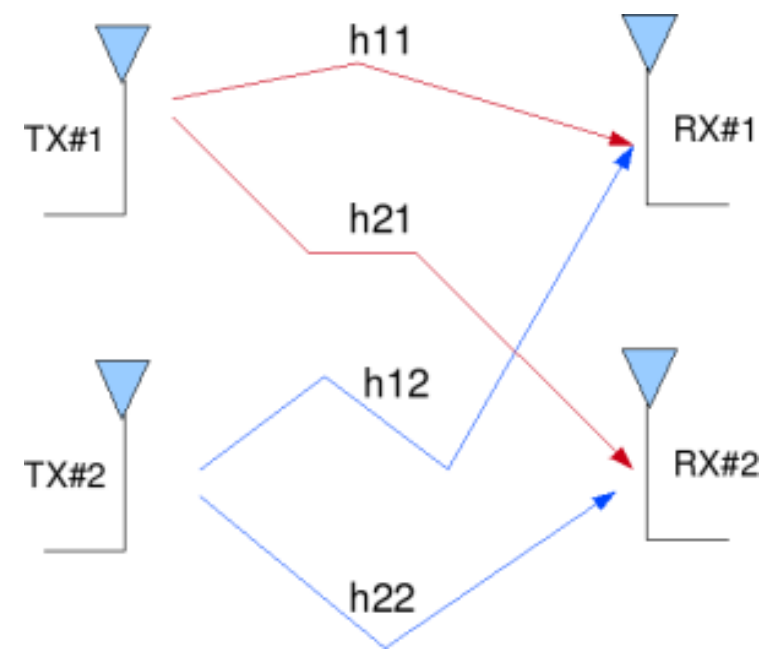

Fig.6 Transmit 2 Receive $(2 \times 2)$ MIMO channel

\section{Other Assumptions}

1. The channel is flat fading - In simple terms, it means that the multipath channel has only one tap. So, the convolution operation reduces to a simple multiplication.

2. The channel experience by each transmit antenna is independent from the channel experienced by other transmit antennas.

3. For the $\mathrm{i}^{\text {th }}$ transmit antenna to $\mathrm{j}^{\text {th }}$ receive antenna, each transmitted symbol gets multiplied by a randomly varying complex number $\mathrm{h}_{\mathrm{j}, \mathrm{i}}$. As the channel under consideration is a Rayleigh channel, the real and imaginary parts of $\mathrm{h}_{\mathrm{j}, \mathrm{i}}$ are Gaussian distributed having mean ${ }^{\mu} \mathrm{h}_{\mathrm{j}, \mathrm{i}}=0$ and variance $\sigma_{h \mathrm{j}, \mathrm{i}}^{2}=1 / 2$.

4. The channel experienced between each transmit to the receive antenna is independent and randomly varying in time.

5. On the receive antenna, the noise $\mathrm{n}$ has the Gaussian probability density function with

$p(n)=\frac{1}{\sqrt{2 \pi \sigma^{2}}} e^{\frac{-(n-\mu)^{2}}{2 \sigma^{2}}}$ with $\mu=0$ and $\sigma^{2}=\mathrm{N}_{0} / 2$.

6 . The channel $\mathrm{h}_{\mathrm{j}, \mathrm{i}}$ is known at the receiver.

\subsection{Zero forcing (ZF) equalizer for $2 \times 2$ MIMO channel}

Let us now try to understand the math for extracting the two symbols which interfered with each other. In the first time slot, the received signal on the first receive antenna is,

$$
y_{1}=h_{1,1} x_{1}+h_{1,2} x_{2}+n_{1}=\left[h_{1,1} h_{1,2}\right]\left[\begin{array}{l}
x_{1} \\
x_{2}
\end{array}\right]+n_{1} \text {. }
$$

The received signal on the second receive antenna is,

$$
y_{2}=h_{2,1} x_{1}+h_{2,2} x_{2}+n_{2}=\left[h_{2,1} h_{2,2}\right]\left[\begin{array}{l}
x_{1} \\
x_{2}
\end{array}\right]+n_{2} \text {. }
$$

Where

$\mathrm{y}_{1}, \mathrm{y}_{2}$ are the received symbol on the first and second antenna respectively,

$\mathrm{h}_{1,1}$ is the channel from $1^{\text {st }}$ transmit antenna to $1^{\text {st }}$ receive antenna, 
$\mathrm{h}_{1,2}$ is the channel from $2^{\text {nd }}$ transmit antenna to $1^{\text {st }}$ receive antenna,

$\mathrm{h}_{2,1}$ is the channel from $1^{\text {st }}$ transmit antenna to $2^{\text {nd }}$ receive antenna,

$\mathrm{h}_{2,2}$ is the channel from $2^{\text {nd }}$ transmit antenna to $2^{\text {nd }}$ receive antenna,

$\mathrm{x}_{1}, \mathrm{x}_{2}$ are the transmitted symbols and

$\mathrm{n}_{1}, \mathrm{n}_{2}$ is the noise on $1^{\text {st }}, 2^{\text {nd }}$ receiver antennas.

We assume that the receiver knows $h_{1.1}, h_{1,2}, h_{2,1}$ and $h_{2,2}$. The receiver also knows $y_{1}$ and $y_{2}$.

The unknowns are $x_{1}$ and $x_{2}$. We have two equations and two unknowns.

For convenience, the above equation can be represented in matrix notation as follows:

$$
\left[\begin{array}{l}
y_{1} \\
y_{2}
\end{array}\right]=\left[\begin{array}{ll}
h_{1,1} & h_{1,2} \\
h_{2,1} & h_{2,2}
\end{array}\right]\left[\begin{array}{l}
x_{1} \\
x_{2}
\end{array}\right]+\left[\begin{array}{l}
n_{1} \\
n_{2}
\end{array}\right]
$$

Equivalently,

$\mathrm{y}=\mathrm{Hx}+\mathrm{n}$

To solve for $\mathrm{x}$, we know that we need to find a matrix $\mathrm{W}$ which satisfies $\mathrm{WH}=\mathrm{I}$. The Zero

Forcing (ZF) linear detector for meeting this constraint is given by,

$\mathrm{W}=\left(\mathrm{H}^{\mathrm{H}} \mathrm{H}\right)^{-1} \mathrm{H}^{\mathrm{H}}$

This matrix is also known as the pseudo inverse for a general $\mathrm{m} \mathrm{x} \mathrm{n}$ matrix.

The term,

$$
\boldsymbol{H}^{\boldsymbol{H}} \boldsymbol{H}=\left[\begin{array}{cc}
h_{1,1}^{*} & h_{2,1}^{*} \\
h_{1,2}^{*} & h_{2,2}^{*}
\end{array}\right]\left[\begin{array}{cc}
h_{1,1} & h_{1,2} \\
h_{2,1} & h_{2,2}
\end{array}\right]=\left[\begin{array}{cc}
\left|h_{1,1}\right|^{2}+\left|h_{2,1}\right|^{2} & h_{1,1}^{*} h_{1,2}+h_{2,1}^{*} h_{2,2} \\
h_{1,2}^{*} h_{1,1}+h_{2,2}^{*} h_{2,1} & \left|h_{1,2}\right|^{2}+\left|h_{2,2}\right|^{2}
\end{array}\right]
$$

\subsection{BER with $\mathrm{ZF}$ equalizer with $2 \times 2$ MIMO}

Note that the off diagonal terms in the matrix $\mathrm{H}^{\mathrm{H}} \mathrm{H}$ are not zero. Because the off diagonal terms are not zero, the zero forcing equalizer tries to null out the interfering terms when performing the equalization, i.e. when solving for $\mathrm{x}_{1}$ the interference from $\mathrm{x}_{2}$ is tried to be nulled and vice versa. While doing so, there can be amplification of noise. Hence Zero Forcing equalizer is not the best possible equalizer to do the job. However, it is simple and reasonably easy to implement.

Further, it can be seen that, following zero forcing equalization, the channel for symbol transmitted from each spatial dimension (space is antenna) is a like a $1 \times 1$ Rayleigh fading channel. Hence the BER for $2 \times 2$ MIMO channel in Rayleigh fading with Zero Forcing equalization is same as the BER derived for a $1 \times 1$ channel in Rayleigh fading.

For BPSK modulation in Rayleigh fading channel, the bit error rate is derived as, 
$P_{b}=\frac{1}{2}\left(1-\sqrt{\frac{\left(E_{b} / N_{0}\right)}{\left(E_{b} / N_{0}\right)+1}}\right)$.

\section{Simulation Algorithm}

(a) Generate random binary sequence of +1 's and -1 's.

(b) Group them into pair of two symbols and send two symbols in one time slot

(c) Multiply the symbols with the channel and then add white Gaussian noise.

(d) Equalize the received symbols

(e) Perform hard decision decoding and count the bit errors

(f) Repeat for multiple values of $\mathrm{E}_{\mathrm{b}} / \mathrm{N}_{0}$ and plot the simulation and theoretical results.

\subsection{MIMO with Zero Forcing Successive Interference Cancellation equalizer}

We will try to improve the bit error rate performance by trying out Successive Interference Cancellation (SIC); assuming that the channel is a flat fading Rayleigh multipath channel and the modulation is BPSK.

\subsection{Zero Forcing with Successive Interference Cancellation (ZF-SIC)}

Using the Zero Forcing (ZF) equalization approach described above, the receiver can obtain an estimate of the two transmitted symbols $\mathrm{x}_{1}$, $\mathrm{x}_{2}$, i.e.

$$
\left[\begin{array}{l}
\widehat{x}_{1} \\
\widehat{x}_{2}
\end{array}\right]=\left(\boldsymbol{H}^{H} \boldsymbol{H}\right)^{-1} \boldsymbol{H} \boldsymbol{H}\left[\begin{array}{l}
y_{1} \\
y_{2}
\end{array}\right]
$$

Take one of the estimated symbols (for example $\widehat{x}_{2}$ ) and subtract its effect from the received vector $\mathrm{y}_{1}$ and $\mathrm{y}_{2}$, i.e.

$$
\left[\begin{array}{l}
r_{1} \\
r_{2}
\end{array}\right]=\left[\begin{array}{ll}
y_{1}-h_{1,2} \widehat{x}_{2} \\
y_{2}-h_{2,2} \widehat{x}_{2}
\end{array}\right]=\left[\begin{array}{ll}
h_{1,1} x_{1}+n_{1} \\
h_{2,1} x_{1}+n_{2}
\end{array}\right]
$$

Expressing in matrix notation,

$$
\left[\begin{array}{l}
r_{1} \\
r_{2}
\end{array}\right]=\left[\begin{array}{l}
h_{1,1} \\
h_{2,1}
\end{array}\right] x_{1}+\left[\begin{array}{l}
n_{1} \\
n_{2}
\end{array}\right]
$$

$\mathrm{r}=\mathrm{hx}_{1}+\mathrm{n}$

The above equation is same as equation obtained for receive diversity case. Optimal way of combining the information from multiple copies of the received symbols in receive diversity case is to apply Maximal Ratio Combining (MRC).

The equalized symbol is,

$\widehat{x}_{1}=\frac{\hbar^{X}{ }_{F}}{h^{K} h}$ 
This forms the simple explanation for Zero Forcing Equalizer with Successive Interference Cancellation (ZF-SIC) approach.

\section{Simulation Algorithm}

(a) Generate random binary sequence of +1 's and -1 's.

(b) Group them into pair of two symbols and send two symbols in one time slot

(c) Multiply the symbols with the channel and then add white Gaussian noise.

(d) Equalize the received symbols with Zero Forcing criterion

(e) Take the symbol from the second spatial dimension, subtract from the received symbol

(f) Perform Maximal Ratio Combining for equalizing the new received symbol

(g) Perform hard decision decoding and count the bit errors

(h) Repeat for multiple values of $\mathrm{E}_{\mathrm{b}} / \mathrm{N}_{0}$ and plot the simulation and theoretical results.

\section{SIMULATION RESULTS}

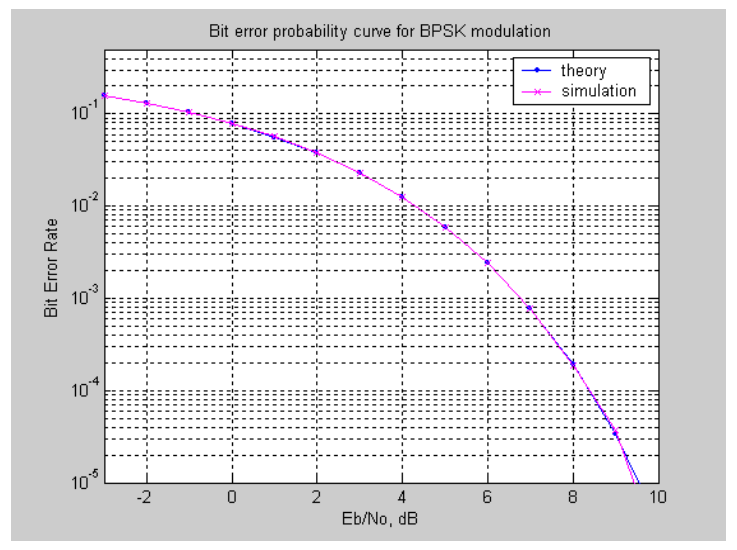

Fig.7 BER versus S/N for BPSK modulation

Fig. 7 shows a computer simulation for BER versus $\mathrm{S} / \mathrm{N}$ ratio of 2 transmitting antennas and 2 receiving antennas in MIMO using BPSK modulation. The BER decreases from $10^{-1}$ for SNR from 0 to $10 \mathrm{~dB}$. Similarly the simulation results for ZF-SIC and MMSE-SIC equalizer are shown in fig. 8 and fig.9 respectively.

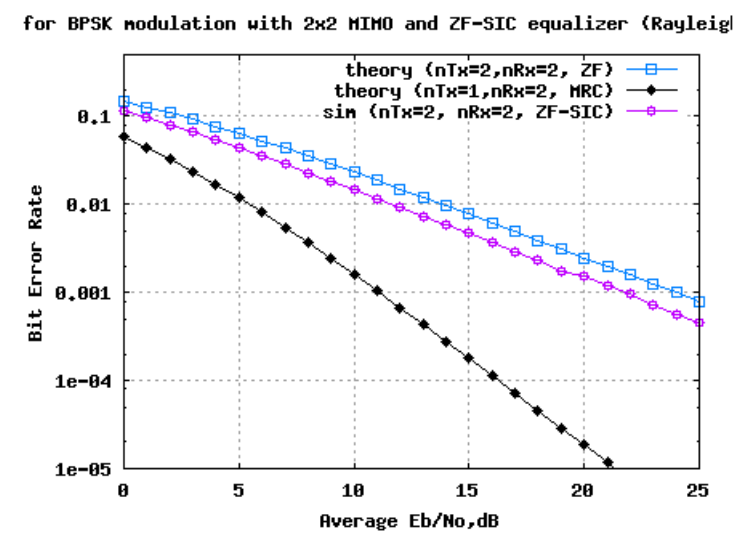

Fig.8 BPSK modulation with 2*2 MIMO and ZF-SIC Equalizer 


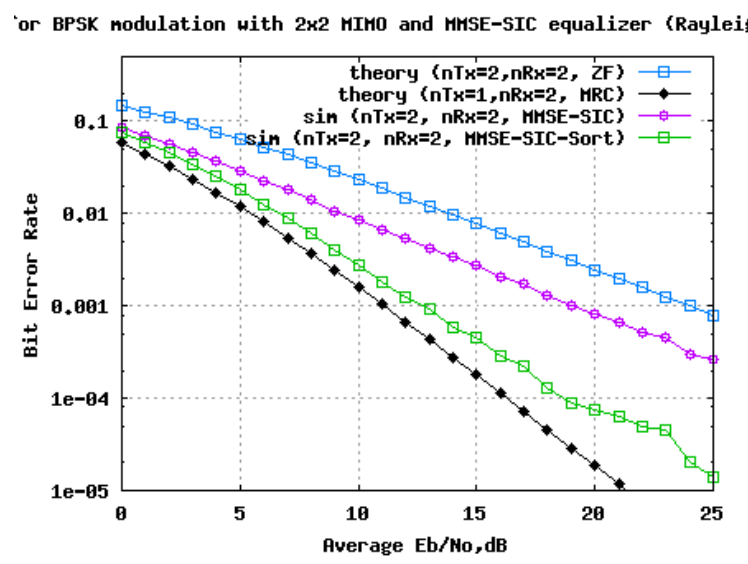

Fig.9 BPSK modulation with $2 * 2$ MIMO and MMSE-SIC equalizer

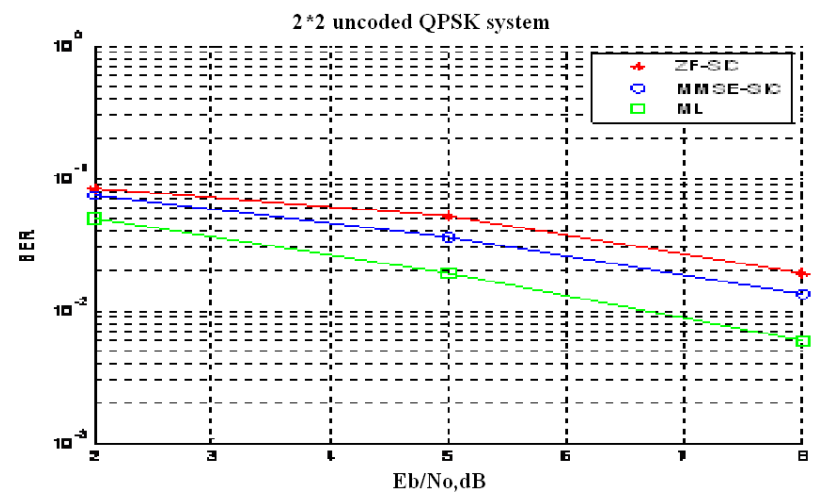

Fig.10 Comparison of ZF-SIC, MMSE-SIC and ML equalizer

Fig. 10 represents a computer simulation comparing the BER performance for ZF-SIC, MMSESIC and ML equalizer. The Mat lab specifications of MIMO system are shown below.

1. $2 * 2$ MIMO system: 2 transmitter and 2 receiver antennas.

2. QPSK Modulation technique

3. Number of transmitted bits: 64

4. Number of iterations: 100

5. Signal to noise ratio: [ 2:3:8 ] i.e [2,3,4,5,6,7,8, dB]

Here input binary data is sent through QPSK modulator. The data is taken in the form of symbols. The modulated data is sent through noisy channel and the data get mixed with the noise. The BER with respect to signal to noise ration is plotted using Mat lab. The error rate is goes on reducing from $10^{0}$ to $10^{-1}$ as the signal to noise ratio is from 0 to $8 \mathrm{~d} \mathrm{~B}$. As the number of iterations increases, the error reduces so that BER versus SNR goes on reducing and become negligible. Here the ML Equalizer has BER of less than $10^{-1}$ when compared to ZF-SIC and MMSE-SIC equalizer. Finally we can conclude that ML equalizer is the best one to overcome multipath fading in cellular system.

\section{CONCLUSION}

MIMO systems are gaining much more attention and efforts in wireless communication research due to their potential to increase considerable capacity in mobile cellular 
communication. MIMO technology can achieve both multiplexing gain \& diversity gain $\&$ thus promises significant improvements in terms of spectral efficiency \& link reliability. MIMO technique is a promising technique that can be used in the next generation mobile communications. Lastly the interference cancellation technologies can suppress the co- channel interference effectively.

\section{REFERENCES}

[1] I.E.Telator Capacity of multi-antenna Gaussian -Channels. .European Transaction on Telecommunications, 10 (6), .Nov 1999.pp.585-595.

[2] Foschini, G.J., and Gans, M.J.: On limits of wireless communications in a fading environment when using multiple antennas, Wireless Personal Communication, 1998, pp. 311-335.

[3] V.Tarokh, H Jafarkhani, A R Calderbank. Space-time Block Codes from Orthogonal Design. IEEE Transaction on Information Theory, July 1999.pp 1456-1467.

[4] E G Larsson, P Storca. Space-time Block Coding for Wireless Communication, Cambridge University Press, Cambridge,UK, 2003.

[5] A. Molisch, wireless communications. Wiley-IEEE press 2005.

[6] G. Tsoulos, MIMO system technology for wireless communication CRC press, 2006.

[7] Vucetic, B., and Yuan, J.: Space-time coding, Jhon Wiley and Sons Ltd, Chichester, U.K, 2003.

[8] Alamouti, S.M.,: A simple transmit diversity technique for wireless communications, IEEE Journal, Select Areas in Communication, 1998, pp. 1451-1458.

[9] David Gesbert, Mansoor Shafi, Da-Shan Shiu, Peter J.Smith, Ayman Naguib. From Theory to Practice, An Overview of MIMO Space-time Coded Wireless System, IEEE Journal on selected areas in Communication, vol21 Issue 3, April 2003,pp.281-298.

[10] Vahid Tarokh, Hamid Jafarkhani, Robert Calderbank, Space-time Block Coding for Wireless Communication: Performance Results, Journal on Selected areas in Communication, Vol. 17, No 3, March 1999.

1. Nazia Parveen received the B.E.,degree in Electronics \& communication in 1993 and M.E.,degree in Communication Systems from Gulbarga university in 1998. She is currently pursuing Ph.D from JNTU., Hyderabad, India. She is working as Associate professor, Dept. of Electronics and communication Engineering, Muffakham Jah College of Engineering and technology, Hyderabad. She has published three papers in international and national conferences. Her research interest includes MIMO wireless communication system, Optical fiber communication and Satellite communication. She is life member of IETE.

2. Dr.D.S.Venketeswarlu received his B.E from Andhra University in 1960, Andhra Pradesh, and M.E from Indian Institute of Science, Bangalore, in 1962, PhD from University of Southampton, UK, in 1967. He has about 13 years industrial experience and about 38 years academic and R\&D experience. He has published about 60 papers in peer reviewed journals (National and International) and presented papers in various National and International conferences. 